\title{
Einstein's action and the harmonic gauge in terms of Newtonian fields
}

\author{
Barak Kol \\ Racah Institute of Physics, Hebrew University \\ Jerusalem 91904, Israel \\ barak_kol@phys.huji.ac.il

\section{Michael Smolkin} \\ Perimeter Institute for Theoretical Physics \\ Waterloo, Ontario N2L 2Y5, Canada \\ msmolkin@perimeterinstitute.ca
}

\begin{abstract}
The "Newtonian" or non-relativistic decomposition of Einstein's gravitational field is useful in the post-Newtonian approximation. We obtain the full non-quadratic Einstein-Hilbert action in terms of these fields as well as the harmonic gauge fixing term and find fairly simple expressions. We discuss alternatives to the harmonic gauge.
\end{abstract}




\section{Contents}

1. Introduction 1

2. NRG fields 3

3. NRG action

3.1 Massive Kaluza-Klein action 6

4. The gauge

4.1 Harmonic gauge fixing term 8

4.2 Comparing gauges for the PN limit 8

A. Deriving the NRG action 12

B. NRG action expanded 14

\section{Introduction}

Newton's universal law of gravitation [1] can be expressed through the action of a (static) gravitational potential $\phi$

$$
S=-\frac{1}{8 \pi G} \int d t d^{3} x(\vec{\nabla} \phi)^{2}
$$

together with its coupling to massive objects $\int d t\left(\frac{m}{2} \dot{\vec{r}}^{2}-m \phi(\vec{r})\right)$.

In Einstein's theory of gravity (General Relativity, GR) [2] the gravitational field is promoted to a space-time metric $g_{\mu \nu}$ subject to the Einstein-Hilbert action

$$
S_{E H}=-\frac{1}{16 \pi G} \int \sqrt{-g} d^{4} x R[g],
$$

where the overall sign in (1.2) reflects the signature convention for $g_{\mu \nu}$ which we take to be time-like. The coupling to massive objects becomes $-m \int d \tau$, where $x^{\mu}(\tau)$ is the particle's trajectory, the proper time is defined by $d \tau^{2}:=g_{\mu \nu} d x^{\mu} d x^{\nu}$, and for clarity we used $c=1$ units.

In the Newtonian limit Einstein's gravity reduces to Newton's. Yet, Einstein's theory calls for a 10 component field, while Newton's theory has only one. A natural question arises: what is the physical role of the other metric components in the Newtonian limit?

In 2007 we proposed a change of variables from the metric to certain non-relativistic gravitational (NRG) fields based on a temporal Kaluza-Klein reduction. One of them can be identified with the Newtonian potential $\phi$, and the others also have a role, even 
if subleading, in this limit, as we review below, and hence they can be called "Newtonian". However, the action (and hence also the equations of motion) were not fully known. Determining the full action including gauge fixing terms is the objective of this paper.

We carried the computation using a non-orthonormal frame within Cartan's method, namely a hybrid method which incorporates both a non-trivial frame and a non-trivial metric. The process turned out to be related to existing work in the Kaluza-Klein literature 27, 28] but it was not used so far in the gravitational post-Newtonian context to the best of our knowledge. We also computed the harmonic gauge fixing term for these fields, thereby completing the evaluation of the total action.

Background. The Newtonian limit of Einstein's gravity was an issue since its very beginning [2, 3], continued with [4] and developed into [5]. The interest further arose in the last three decades with the development of the Post-Newtonian (PN) approximation, see [6, 7] for recent reviews and [8, 9] for some earlier work. The central application of the PostNewtonian approximation is the analysis of the two body problem in GR and the resulting gravitational wave signature. This problem is crucial for the global effort to directly detect gravitational waves [10] and eventually interpret their signals.

In 2004 the effective field theory (EFT) approach to GR [11] was put forward. This approach borrows from the ideas of effective quantum field theories. It is based on a hierarchy of scales in the problem and essentially replaces the traditional method of dealing with finite size objects through matched asymptotic expansion by introducing instead effective interactions of point particles with their background. See [12] for early precursors of the EFT method.

In [13, 14] we introduced the NRG fields ${ }^{1}$ and used them to give what is probably the shortest derivation of the leading post-Newtonian correction, known as the Einstein-InfeldHoffmann interaction 画. Their utility received strong support from [16] who reproduced the $2 \mathrm{PN}$ effective action (see [6] and references therein) through the EFT approach. The authors of [16] compared the NRG fields against the standard metric, and found the NRG fields to be better suited for the calculational purpose. After the first arXiv version of this paper appeared further support was received when the computation was successfully extended to reproduce order 3PN using an impressive computerized calculation [17]. The computations in [14, 16, 17] required the evaluation of certain terms in the NRG action and those were evaluated in a perturbative weak field expansion and sometimes with the help of computerized computation. The full and concise non-perturbative expressions presented here allows to readily expand and read off the gravitational vertices for the PN perturbation theory and it should be useful for further research including advancing the post-Newtonian state-of-the-art and reaching order $4 P N$ (which is not available yet in any method), a goal announced in [17]. In such higher orders the recursive diagrammatic relations of [18] should be helpful as well. Related interesting and relatively recent work on PN and/ or EFT appeared in [19].

This paper is organized as follows. In section 2 we review the definition of NRG fields.

\footnotetext{
${ }^{1}$ See [9, 15] for early precursors of the NRG fields.
} 
In section 3 we present the Einstein-Hilbert action in terms of these fields (the derivation is relegated to an appendix). In section 1 we discuss possible gauge choices from the PN perspective and compute the harmonic gauge fixing term in NRG fields.

\section{NRG fields}

The non-relativistic limit is defined as the limit of slow velocities (relative to the speed of light). As a zeroth approximation we consider the stronger time-independent (stationary) limit. In this limit it is natural to perform a temporal Kaluza-Klein (KK) dimensional reduction [13, 14] (the original and standard KK reduction 20] was spatial, of course)

$$
d s^{2}=e^{2 \phi}\left(d t-A_{i} d x^{i}\right)^{2}-e^{-2 \phi} \gamma_{i j} d x^{i} d x^{j}
$$

This relation defines a change of variables from $g_{\mu \nu}$ to $\left(\phi, A_{i}, \gamma_{i j}\right), i, j=1,2,3$ which we call "non-relativistic gravitational fields", or in short "NRG-fields".

The action, when translated into NRG-fields and within the stationary limit becomes

$$
S=-\frac{1}{16 \pi G} \int d t d^{3} x \sqrt{\gamma}\left[-R[\gamma]+2|\vec{\nabla} \phi|^{2}-\frac{1}{4} e^{4 \phi} F^{2}\right],
$$

where $|\vec{\nabla} \phi|^{2}=\gamma^{i j} \partial_{i} \phi \partial_{j} \phi$ and $F^{2}$ is conventionally defined by $F^{2}=F_{i j} F^{i j}, \quad F_{i j}=$ $\partial_{i} A_{j}-\partial_{j} A_{i}$.

The above-defined NRG fields (2.1) resemble the definition of the well-known ADM fields [21] (see also the review 222 and references therein) given by $d s^{2}=N^{2} d t^{2}-$ $\gamma_{A D M i j}\left(d x^{i}+N^{i} d t\right)\left(d x^{j}+N^{j} d t\right)$, where $N, N^{i}$ are the lapse and shift. Moreover, for $A_{i}=0=N^{i}$ the definitions coincide as they do at linear order for all fields (after a suitable Weyl-rescaling of the ADM fields). However, there is a marked difference between the two: the ADM fields are designed for the initial value problem and the time evolution of the metric, while NRG fields are designed for the post-Newtonian evolution of say a two-body problem. The NRG fields are defined by a temporal KK reduction, while ADM is nothing but a spatial KK reduction (in order to focus on the time evolution), namely in NRG the shift is applied to time and in ADM to space. More concretely this means that NRG fields transform nicely under time-independent coordinate reparameterizations, while ADM transforms nicely in the space-independent case. So in this sense ADM and NRG are sort of opposites actually.

After the first arXiv version of this paper appeared a detailed and practical comparison between NRG, ADM and a modified ADM fields was made 23] in the PN context and NRG was shown to be the more economical field definition starting at order $2 \mathrm{PN}$.

It is interesting that even though the Kaluza-Klein (KK) ansatz was available since 1921, it was not applied in full to the post-Newtonian approximation before [13, 14] as far as we are aware. One possible reason is that the usual KK procedure is for a compact space-like dimension while here it is applied to time.

Physical meaning of NRG fields $\phi, A_{i}, \gamma_{i j}$. Comparing the kinetic term for $\phi$ in the action (2.2), with the Newtonian field action (1.1) together with the respective interaction 
terms we find it natural to identify $\phi$ with the Newtonian potential. To leading order around flat-space time (Newtonian limit) this definition coincides with the accepted definitions of the Newtonian potential $\phi_{N}$ in the literature, for instance $g_{00} \simeq 1+2 \phi_{N}$. Due to the nearly stationary nature of the Newtonian limit, we find $\phi$ to be a good way to extend this definition beyond the linear order.

The vector potential $\vec{A}$ has an action which apart for an overall sign resembles the magnetic part of the $4 \mathrm{~d}$ Maxwell action, and accordingly it is natural to call $F$ the gravitomagnetic field and call $\vec{A}$ the gravito-magnetic vector potential. This name originates in a certain similarity between gravity and electro-magnetism. The strong similarity between Newton's gravitational force and Coulomb's static electrical force, together with the observation that the transition from electro-statics to electro-dynamics requires to supplement the scalar electric potential by a vector potential, promoted already in the 19th century suggestions to add a vector potential to the gravitational degrees of freedom. In fact, it is known how to obtain such a vector potential in the weak gravity/ Post-Newtonian approximation to GR, a point of view known as "Gravito-Electro-Magnetism (GEM)" (see for example [24, 15]) and references within). Just like in electromagnetism the vector potential couples at leading order to the charged current, which in gravity is the mass current, namely $S \supset m \int d t \vec{v} \cdot \vec{A}$, and it is responsible for a gravito-magnetic force between two mass currents. A spinning object consists of a dipole of mass current and accordingly it couples universally to the magnetic field $\vec{B}=\vec{\nabla} \times \vec{A}$ through $S \supset \frac{1}{2} \int d t \vec{J} \cdot \vec{B}$. We note the reversed sign of the kinetic term for $F$. This is directly related to the fact that in gravity both the current-current and the spin-spin force have an opposite sign relative to electro-dynamics, and so "north poles attract" [25].

Finally, starting with order $2 \mathrm{PN}$ we must also account for the 3 -metric tensor $\gamma_{i j}$ which comes with a standard Einstein-Hilbert action in 3d (this is achieved through the Weyl rescaling factor in front of $\gamma_{i j}$ in the ansatz). This field has no electromagnetic analogue.

\section{NRG action}

In this section we go beyond the stationary approximation of the last section and derive the full, time-dependent action for the NRG fields. For greater generality we work in an arbitrary space-time dimension $d$. We perform the change of variables in two steps. In the first we replace

$$
g_{\mu \nu} \rightarrow\left(\phi, \vec{A}, \widetilde{\gamma}_{i j}\right)
$$

and in the second step

$$
\widetilde{\gamma}_{i j} \rightarrow \gamma_{i j}
$$

The first step is achieved through a dimensional reduction

$$
d s^{2}=e^{2 \phi}\left(d t-A_{i} d x^{i}\right)^{2}-\widetilde{\gamma}_{i j} d x^{i} d x^{j}
$$


The Einstein-Hilbert action $S=-1 /(16 \pi G) \int R d V$ becomes

$$
\begin{aligned}
S= & -\frac{1}{16 \pi G} \int e^{\phi} \sqrt{\widetilde{\gamma}} d^{d-1} x d t \\
& \left\{-\frac{1}{4} e^{2 \phi} \bar{F}^{2}-\frac{1}{4} e^{-2 \phi}\left(\dot{\widetilde{\gamma}}_{i j} \dot{\widetilde{\gamma}}_{k l} \widetilde{\gamma}^{i k} \widetilde{\gamma}^{j l}-\left(\widetilde{\gamma}^{i j} \dot{\widetilde{\gamma}}_{i j}\right)^{2}\right)-\bar{R}[\widetilde{\gamma}]\right\}
\end{aligned}
$$

where we define

$$
\begin{aligned}
& D_{i}:=\partial_{i}+A_{i} \partial_{t} \\
& \bar{F}_{i j}:=D_{i} A_{j}-D_{j} A_{i}=F_{i j}+A_{i} \dot{A}_{j}-A_{j} \dot{A}_{i},
\end{aligned}
$$

a dot denotes a time derivative and $\bar{R}[\widetilde{\gamma}]$ denotes the Ricci scalar of the spatial metric $\widetilde{\gamma}_{i j}$ where the derivatives in its expression are replaced everywhere as follows $\partial_{i} \rightarrow D_{i}$. Borrowing notation from the Mathematica software this definition can be stated by

$$
\bar{R}[\widetilde{\gamma}]:=R[\widetilde{\gamma}] / . \partial_{i} \rightarrow D_{i}
$$

Note the following alternative forms for two of the terms in 3.3): $\dot{\tilde{\gamma}}_{i j} \dot{\tilde{\gamma}}_{k l} \widetilde{\gamma}^{i k} \widetilde{\gamma}^{j l}=$ $-\dot{\widetilde{\gamma}}^{i j} \dot{\widetilde{\gamma}}_{i j}$ and $\widetilde{\gamma}^{i j} \dot{\widetilde{\gamma}}_{i j}=\partial_{t} \log \operatorname{det} \widetilde{\gamma}$

The action (3.3) for the dimensionally reduced fields (3.2) is the essential step towards incorporating time dependence into the action, and we find that it leaves quite a compact result: the $\bar{F}^{2}, \bar{R}$ terms generalize appropriately the well-known stationary action, while the remaining term is a kinetic term for $\gamma_{i j}$ which defines an associated metric on field space, namely on the space of metrics, and it coincides with one of the deWitt metrics [26]. In order to compute the action we used a non-orthonormal frame within Cartan's method, namely a hybrid method which incorporates both a non-trivial frame and a non-trivial metric (see appendix A).

The second step is a Weyl rescaling

$$
\widetilde{\gamma}_{i j}=e^{-2 \phi / \tilde{d}} \gamma_{i j}
$$

where we use a shorthand notation

$$
\tilde{d}:=d-3 .
$$

Altogether the metric reads now

$$
d s^{2}=e^{2 \phi}\left(d t-A_{i} d x^{i}\right)^{2}-e^{-2 \phi / \tilde{d}} \gamma_{i j} d x^{i} d x^{j}
$$

which agrees with (2.1) for $d=4$. The Weyl rescaling is designed such that the stationary action for $\gamma_{i j}$ will be the standard Einstein-Hilbert action, without the $e^{\phi}$ prefactor present in (3.3). We denote the perturbation of the spatial metric $\gamma_{i j}$ by

$$
\sigma_{i j}:=\gamma_{i j}-\delta_{i j}
$$


Substituting into the action we obtain our main result, the fully non-linear and timedependent Einstein-Hilbert action for the NRG fields

$$
\begin{aligned}
S= & -\frac{1}{16 \pi G} \int \sqrt{\gamma} d^{d-1} x d t \\
& \left\{\left(1+\frac{1}{\tilde{d}}\right)\left|D_{i} \phi\right|^{2}-\frac{1}{4} e^{2(1+1 / \tilde{d}) \phi} \bar{F}^{2}-\bar{R}[\gamma]\right. \\
& +2(1+1 / \tilde{d}) \gamma^{i j} \dot{A}_{i} D_{j} \phi \\
& -\frac{1}{4} e^{-2(1+1 / \tilde{d}) \phi}\left(\dot{\gamma}_{i j} \dot{\gamma}_{k l} \gamma^{i k} \gamma^{j l}-\left(\gamma^{i j} \dot{\gamma}_{i j}\right)^{2}\right) \\
& \left.-e^{-2(1+1 / \tilde{d}) \phi}\left(\left(1+\frac{1}{\tilde{d}}\right) \dot{\phi} \gamma^{i j} \dot{\gamma}_{i j}-\frac{(\tilde{d}+2)(\tilde{d}+1)}{\tilde{d}^{2}} \dot{\phi}^{2}\right)\right\}
\end{aligned}
$$

In particular for $d=4$ space-time dimensions the full NRG or Post-Newtonian action is

$$
\begin{aligned}
S= & -\frac{1}{16 \pi G} \int \sqrt{\gamma} d^{3} x d t \\
& \left\{2\left|D_{i} \phi\right|^{2}-\frac{1}{4} e^{4 \phi} \bar{F}^{2}-\bar{R}[\gamma]+4 \gamma^{i j} \dot{A}_{i} D_{j} \phi\right. \\
& \left.-\frac{1}{4} e^{-4 \phi}\left(\dot{\gamma}_{i j} \dot{\gamma}_{k l} \gamma^{i k} \gamma^{j l}-\left(\gamma^{i j} \dot{\gamma}_{i j}\right)^{2}\right)-e^{-4 \phi}\left(2 \dot{\phi} \gamma^{i j} \dot{\gamma}_{i j}-6 \dot{\phi}^{2}\right)\right\} .
\end{aligned}
$$

We tested our expression for the action in several limits. In the stationary limit it reduces correctly to (2.2). A strong test is provided by the sector with two time derivatives. Since it contains no spatial derivatives, the same sector can be computed by a performing dimensional reduction over space, defining fields similar to the ADM decomposition. The action is given by an the analogue of (2.2) which is then followed by a change of variables to NRG. The result matches with the expansion of the two time derivatives of (3.3) given by $(\mathrm{B} .3)$.

The expression for the action, together with the gauge fixing term (4.3) conveniently encodes the Feynman rules for the diagrammatic computation of the two-body effective action in the EFT method [11, 14, 16, 18]. The relevant terms up to order 2PN include the quadratic time-dependent vertices

$$
\dot{\phi}^{2}, \dot{A}^{2}
$$

and the non-quadratic vertices of the form

$$
\phi \dot{\phi}^{2}, \nabla \phi \dot{\phi} A, \phi(\nabla A)^{2}, \sigma_{i j} \partial_{i} \phi \partial_{j} \phi
$$

Our expression, together with the recursive relations of [18] should be useful for computing higher PN orders.

\subsection{Massive Kaluza-Klein action}

Suppose we take the KK direction to be space-like (as in the original KK idea) and denote it by $z$. Then our action (3.10) is related to the full action including $z$-dependent fields, 
namely the massive modes. It turns out that this action is essentially known in the literature: the $4+1$ pre-Weyl rescaling action in compact form (analogous to our (3.3)) was found by Aulakh and Sahdev [27] and an expanded 4+1 post Weyl rescaling expression (analogous to our (B.1) ) was found by Maheshwari [28] (see also the reviews [29]). Below we illustrate how to use our result to obtain the full $4+1$ action after Weyl rescaling and in compact form.

The metric ansatz becomes

$$
d s^{2}=e^{-2 \phi / \tilde{d}} \gamma_{\mu \nu} d x^{\mu} d x^{\nu}-e^{2 \phi}\left(d z-A_{\mu} d x^{\mu}\right)^{2}
$$

where $\mu, \nu=0,1, \ldots, d-2$. We observe that the substitution

$$
t \leftrightarrow z
$$

amounts to reversing the Ricci scalar (since it reverses the metric in the non-orthonormal frame)

$$
R\left[g_{\mu \nu}\right] \rightarrow-R\left[g_{\mu \nu}\right] .
$$

This allows us to obtain the action by reversing (3.10). For concreteness let us present the form of the KK action for $d=5$

$$
\begin{aligned}
S= & \frac{1}{16 \pi G} \int \sqrt{-\gamma} d^{3} x d t d z \\
& \left\{\frac{3}{2}\left|D_{\mu} \phi\right|^{2}-\frac{1}{4} e^{3 \phi} \bar{F}^{2}-\bar{R}[\gamma]+3 \gamma^{\mu \nu} D_{\mu} \phi \partial_{z} A_{\nu}\right. \\
& \left.-\frac{1}{4} e^{-3 \phi}\left(\gamma^{\mu \rho} \gamma^{\nu \sigma}\left(\partial_{z} \gamma_{\mu \nu}\right)\left(\partial_{z} \gamma_{\rho \sigma}\right)-\left(\gamma^{\mu \nu} \partial_{z} \gamma_{\mu \nu}\right)^{2}\right)-e^{-3 \phi}\left(\frac{3}{2}\left(\gamma^{\mu \nu} \partial_{z} \gamma_{\mu \nu}\right) \partial_{z} \phi-3\left(\partial_{z} \phi\right)^{2}\right)\right\} .
\end{aligned}
$$

This expression could be useful for studying non-linear interactions involving massive KK modes.

\section{The gauge}

In computing the two-body effective action we are free to choose the gauge (choice of coordinates). It affects both the length of the calculation as well as the end result since the effective action depends on the coordinate location of the two bodies and as such is not gauge invariant.

The harmonic gauge is used throughout the PN literature. It is defined by

$$
0=\Gamma^{\mu}:=\Gamma_{\nu \rho}^{\mu} g^{\nu \rho} \equiv-\frac{1}{\sqrt{-g}} \partial_{\nu}\left(\sqrt{-g} g^{\mu \nu}\right)
$$

where $\Gamma_{\nu \rho}^{\mu}$ is the Christoffel symbol for the space-time metric $g$. The corresponding gauge fixing action is taken to be

$$
S_{G F}=\frac{1}{32 \pi G} \int d^{d} x \sqrt{g} g_{\mu \nu} \Gamma^{\mu} \Gamma^{\nu}
$$


In the first subsection we evaluate (4.2) in NRG fields (3.8). In the second subsection we critically discuss the rationale for choosing the harmonic gauge. We note that the harmonic gauge is special for being covariant under Lorentz transformations, while the PN approximation breaks the symmetry between time and space and therefore choosing the harmonic gauge is not obvious. We proceed to analyze this question explicitly order by order in the PN expansion up to $2 \mathrm{PN}$. We summarize our results and discuss them at the end of that subsection.

\subsection{Harmonic gauge fixing term}

In order to express the harmonic gauge fixing term (4.2) in terms of NRG fields we find in appendix $\mathrm{A}$ how to express $\Gamma_{\mu}$ in terms of a frame and a frame metric (A.16). Substituting A.1, A.2 we find

$$
\begin{aligned}
S_{G F}= & \frac{1}{2 \cdot 16 \pi G} \int \sqrt{\gamma} d^{d-1} x d t \\
& \left\{\left(e^{(1+1 / \tilde{d}) \phi} \gamma^{i j} D_{i} A_{j}+2\left(1+\frac{1}{\tilde{d}}\right) e^{-(1+1 / \tilde{d}) \phi} \dot{\phi}-\frac{1}{2} e^{-(1+1 / \tilde{d}) \phi} \partial_{t} \log \gamma\right)^{2}-\left|\bar{\Gamma}_{i}[\gamma]-\dot{A}_{i}\right|^{2}\right\}
\end{aligned}
$$

where we define

$$
\begin{aligned}
\bar{\Gamma}_{i j k}[\gamma] & :=\Gamma_{i j k}[\gamma] / . \partial_{i} \rightarrow D_{i} \\
& =\Gamma_{i j k}[\gamma]+\frac{1}{2}\left(A_{j} \dot{\gamma}_{i k}+A_{k} \dot{\gamma}_{i j}-A_{i} \dot{\gamma}_{j k}\right) .
\end{aligned}
$$

The expression (4.3) was tested by using two additional frame representations of the metric: an orthonormal frame and another which shifts the field $\phi$ from the frame to the metric.

\subsection{Comparing gauges for the $\mathrm{PN}$ limit}

In general, the freedom to choose a gauge could be a blessing provided that it is chosen judiciously. Actually given a specific problem there must be an optimal gauge (simply because the computational cost should be bounded from below and non-zero). Here we are considering the PN approximation, which defines a certain class of problems, meaning that only partial information about the system is known. Accordingly we expect that there would not be a single optimal gauge, but rather an optimal class of gauges where some of the gauge freedom is fixed while some residual gauge freedom remains to fit a given, specific problem.

In this subsection we ask what is the optimal gauge (class) for the post-Newtonian approximation.

The harmonic gauge fixing appears to be standard in the literature. ${ }^{2}$ However, the rationale for that is not clear to us. One of the appealing features of the harmonic gauge (perhaps characterizing it) is its covariance property being a vector under global Lorentz transformations of (asymptotically) flat space-times. Yet, in the PN approximation, Lorentz

\footnotetext{
${ }^{2}$ Sometimes one encounters the closely related linearized harmonic gauge.
} 
invariance is broken to spatial rotations by the choice of a rest frame (choice of time), and hence we proceed to consider a more general class of gauges with less symmetry order by order in the PN expansion.

The Newtonian order. The newtonian field is gauge-invariant to leading, stationary gauge transformations. Accordingly the question of gauge choice is moot.

Quadratic stationary gauge fixing starting from 1PN. In the PN approximation we decompose the gravitational field into the Newtonian potential $\phi$, the gravito-magnetic vector $A_{i}$ and perturbations $\sigma_{i j}$ of the spatial metric tensor $\gamma_{i j}$ (3.8,3.9). The vector appears in the stationary, unperturbed action (2.2) in a Maxwell form, and accordingly the standard Lorentz gauge fixing term must be chosen at the quadratic level

$$
\begin{aligned}
S_{G F} & =\frac{1}{32 \pi G} \int d^{d} x C_{0}^{2} \\
C_{0} & =\vec{\nabla} \cdot \vec{A} .
\end{aligned}
$$

in order to avoid the propagator from mixing the different vector components. This gauge fixing term affects order $1 \mathrm{PN}$ through the diagram of gravito-magnetic interaction (vector exchange).

Similarly $\gamma_{i j}$, the spatial metric has an Einstein-Hilbert action and for simplicity of the propagator we better choose the harmonic gauge (note: only in the spatial directions), at least linearly. This gauge choice shows up first at order $2 \mathrm{PN}$ through the diagram of $\sigma$ exchange.

Quadratic but non-stationary sector starting from 1PN. Before gauge fixing the action includes a term of the form $\dot{\vec{A}} \cdot \vec{\nabla} \phi$ just like the post-Coulomb approximation to electro-magnetism. This term induces a mixing 2-vertex for $\phi, \vec{A}$. A generic gauge fixing term includes also a $\dot{\phi}^{2}$ vertex and hence generically three diagrams contribute: an exchange of $\phi$ with a retardation vertex, a $\phi-A$ exchange diagram and a diagram with a $\phi-A-\phi$ chain.

However, after integration by parts the $\phi-A 2$-vertex is proportional to $\dot{\phi} \vec{\nabla} \vec{A}$ and can be removed by shifting the gauge condition on $\vec{\nabla} \vec{A}$, more specifically by deforming the gauge condition (4.5) to become

$$
C_{0}=\vec{\nabla} \cdot \vec{A}+2\left(1+\frac{1}{\tilde{d}}\right) \dot{\phi} .
$$

This constraint turns out to be part of the harmonic gauge and hence up to order $1 P N$ the harmonic gauge is certainly optimal. We note that just like the electro-magnetic two-body effective action is gauge invariant at any post-Coulomb order, the same holds in the first order of the gravitational case, due to the similarity of the actions up to this order, and hence the sum of the three diagrams is independent of gauge.

Similarly, at order $2 \mathrm{PN}$ with harmonic gauge the diagram of $A$ exchange with a retardation vertex replaces two diagrams with a $\sigma \dot{A}$ mixing which would appear in a generic gauge.

Non-Quadratic gauge fixing starting from order 2PN. We still have freedom to deform the gauge constraint (4.6) with non-linear terms. This freedom can be used to cancel any term in the action which is proportional to the linearized gauge constraint. 


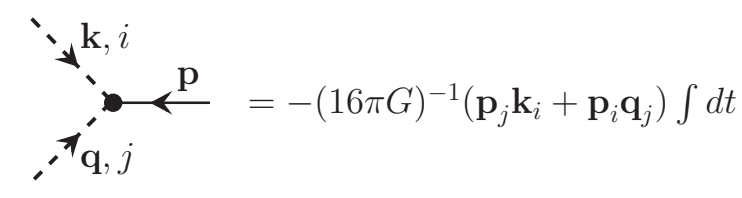

Figure 1: Diagrammatic representation of the new static interaction obtained after gauging away $A^{i} \nabla_{i} \phi \dot{\phi}$ from the Einstein-Hilbert action.

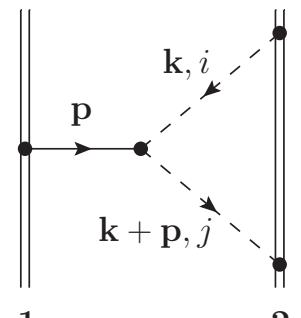

(a)

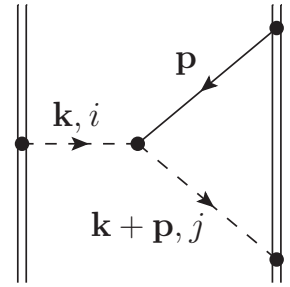

1
2

(b)

Figure 2: New static Feynman diagrams obtained after gauging away $A^{i} \nabla_{i} \phi \dot{\phi}$ from the EinsteinHilbert action. (a) and (b) replace figures 5(l) and 5(k) of Gilmore-Ross respectively.

At order $2 \mathrm{PN}$ the vertex $\vec{\nabla} \phi \dot{\phi} \vec{A}$ appears in two diagrams: $5(\mathrm{~m})$ and $5(\mathrm{n})$ of [16]. We may cancel this term by deforming the gauge condition as follows

$$
C_{0}=\vec{\nabla} \cdot \vec{A}+2\left(1+\frac{1}{\tilde{d}}\right) \dot{\phi}+A^{i} \partial_{i} \phi
$$

At the same time this gauge fixing produces a term of the form $\vec{\nabla} \phi \vec{A} \vec{\nabla} \vec{A}$ which at first sight is already present (see eq. (35) in [16]), however the tensor structure of the new term is different and one must add the static $Y$-type interaction presented in figure 1 .

Computing the value of the terms added to the diagrams we find

$$
\begin{aligned}
& \text { Fig.2 } 2(a)=\frac{d-3}{d-2}\left(16 \pi G m_{2}\right)^{2} \frac{m_{1}}{2} \int d t \int \frac{d^{d-1} k}{(2 \pi)^{d-1}} \frac{d^{d-1} p}{(2 \pi)^{d-1}} \frac{\left(v_{2} \cdot p\right)\left(v_{2} \cdot k\right)}{p^{2} k^{2}(p+k)^{2}} e^{i p \cdot r} \\
& \text { Fig.2 } 2(b)=-\frac{d-3}{d-2}\left(16 \pi G m_{2}\right)^{2} \frac{m_{1}}{2} \int d t \int \frac{d^{d-1} k}{(2 \pi)^{d-1}} \frac{d^{d-1} p}{(2 \pi)^{d-1}} \frac{\left(v_{1} \cdot p\right)\left(v_{2} \cdot p\right)}{p^{2} k^{2}(p+k)^{2}} e^{i k \cdot r} .
\end{aligned}
$$

Integrating over one of the wave numbers and evaluating the resulting Fourier transform by means of (A.2) and (A.10) of [18] respectively, yields (in $\mathrm{d}=4$ )

$$
\begin{aligned}
& \operatorname{Fig} \cdot 2(a)=-2 \frac{G^{2} m_{2}^{2} m_{1}}{r^{2}}\left[\vec{v}_{2}^{2}-2\left(\vec{v}_{2} \cdot \hat{r}\right)^{2}\right], \\
& \operatorname{Fig} \cdot 2(b)=\frac{G^{2} m_{2}^{2} m_{1}}{r^{2}}\left[3\left(\vec{v}_{1} \cdot \hat{r}\right)\left(\vec{v}_{2} \cdot \hat{r}\right)-\left(\vec{v}_{1} \cdot \vec{v}_{2}\right)\right] .
\end{aligned}
$$

We conclude that while in new gauge (4.7) avoids computing two diagrams (fig. $5(\mathrm{~m}, \mathrm{n}$ ) of [16]) it generates two new terms in the diagrams in fig. $5(\mathrm{k}, \mathrm{l})$ which appear to be of 
comparable computational difficulty. Hence it is not clear that the new gauge (4.7) is any better than the harmonic.

Comments about 3PN. At order 3PN log terms appear, reflecting an RG flow 6, 11, 17. The associated divergences are canceled by available redundant terms on the worldline which reflect the choice of coordinates, but not in observables. Presumably this is a price for working with harmonic coordinates. Indeed, the equation for changing the radial coordinate of the Schwarzschild solution from standard to harmonic has log solutions even in asymptotically flat space, and those terms could be essential in background which are not asymptotically flat.

Optimal Post-Newtonian gauge: summary and discussion. We critically analyzed the optimal choice of gauge for the PN approximation. We found that the harmonic gauge is certainly optimal at the level of propagators and 2-vertices (starting with $1 \mathrm{PN}$ ). At $2 \mathrm{PN}$ we found a different gauge which reduces the number of diagrams. However, this does not lead to a reduction in computational difficulty. The reason for that is that each standard Feynman diagram may represent several computational terms corresponding to the positioning of derivatives and to various index structures. A faithful diagrammatic representation of the computational difficulty requires to distinguish vertices of a field from those of its derivatives and also to reflect the index structure. Hence altogether we do not find a gauge which is better than harmonic, at least up to $2 \mathrm{PN}$.

Our analysis suggests that the harmonic gauge could be the optimal PN gauge (in the bulk). In hindsight, the reason for that could be simple. The total PN action is composed of the bulk gravitational action and the interaction with the object's worldline. The computational difficulty is dominated by bulk irreducible loops, which depend on bulk vertices while non-linear world-line vertices contribute only factorizable diagrams (see for example [18]). Hence the choice of gauge depends only on the bulk action which enjoys an increased symmetry (relative to PN). The harmonic gauge can be characterized by its background independence, namely in any background the quadratic action for the fluctuations is given simply by the Lichnerowicz operator. Probably this characterization leads to its advantage also for non-quadratic orders around flat space.

\section{Acknowledgements}

It is a pleasure to thank M. Duff, R. Emparan, G. Gibbons and G. Schäfer for correspondence regarding the manuscript. Special thanks to K. Hinterbichler who attracted our attention to [28].

This research is supported by The Israel Science Foundation grant no 607/05, by the German Israel Cooperation Project grant DIP H.52, and by the Einstein Center at the Hebrew University. Research at Perimeter Institute is supported by the Government of Canada through Industry Canada and by the Province of Ontario through the Ministry of Research \& Innovation. 


\section{A. Deriving the NRG action}

In this section we shall derive (3.3) - the full Einstein-Hilbert action in terms of the dimensionally reduced fields (3.2). We use a non-orthonormal frame within Cartan's method, namely a hybrid method which incorporates both a non-trivial frame and a non-trivial metric, see for example [30].

The dimensionally reduced fields (3.2) define a natural frame of 1-forms

$$
\begin{aligned}
& \theta^{\hat{t}}=e^{\phi}\left(d t-A_{i} d x^{i}\right) \\
& \theta^{\hat{i}}=d x^{i}
\end{aligned}
$$

and a metric

$$
g_{a b}=\operatorname{diag}\left(1,-\widetilde{\gamma}_{i j}\right)
$$

where $a, b=(\hat{t}, \hat{i})$ are frame indices and from hereon we shall replace $\widetilde{\gamma} \rightarrow \gamma$ throughout the derivation for clarity of notation and without risking confusion.

It is convenient to record the inversion of (A.1)

$$
\begin{aligned}
d t & =e^{-\phi} \theta^{\hat{t}}+A_{i} \theta^{\hat{i}} \\
d x^{i} & =\theta^{\hat{i}}
\end{aligned}
$$

as well as the dual basis of vectors

$$
\left\{\begin{array} { l } 
{ e _ { \hat { t } } = e ^ { - \phi } \partial _ { t } } \\
{ e _ { \hat { i } } = \partial _ { i } + A _ { i } \partial _ { t } \equiv D _ { i } }
\end{array} \quad \left\{\begin{array}{l}
\partial_{t}=e^{\phi} e_{\hat{t}} \\
\partial_{i}=e_{\hat{i}}-e^{\phi} A_{i} e_{\hat{t}}
\end{array}\right.\right.
$$

The connection 1-forms $\omega_{b}^{a}$ are the solution of

$$
\begin{aligned}
d \theta^{a}+\omega_{b}^{a} \theta^{b} & =0 \\
\omega_{a b}+\omega_{b a} & =d g_{a b} .
\end{aligned}
$$

In order to solve this system one defines the components of $\omega_{b}^{a}$ to be

$$
\omega_{b}^{a}=\omega_{b c}^{a} \theta^{c}
$$

and these components are given by the sum

$$
\omega_{a b c}=\Sigma_{a b c}+\Gamma_{a b c}
$$

where $\Sigma_{a b c}$ is related to the torsion of the frame, $\Gamma_{a b c}$ is the Christoffel symbol for the frame metric $g_{a b}$ (it is symmetric in $b c$, namely $\Gamma_{a[b c]}=0$ ) and both are defined and computed below.

First one defines a tensor $C_{a b}^{c}$ by

$$
d \theta^{c}+\frac{1}{2} C_{a b}^{c} \theta^{a} \theta^{b}=0 .
$$


$C_{a b}{ }^{c}$ is antisymmetric in $a b$, namely $C_{(a b)}{ }^{c}=0$. In our case one has

$$
\begin{aligned}
& C_{\hat{i} \hat{j}}^{\hat{t}}=e^{\phi} \bar{F}_{i j} \\
& C_{\hat{t} \hat{i}}{ }^{\hat{t}}=D_{i} \phi+\dot{A}_{i}
\end{aligned}
$$

where $\bar{F}, D_{i}$ are defined in (3.4).

$\Sigma_{a b c}$ is defined by

$$
\Sigma_{a b c}=\frac{1}{2}\left(C_{a b c}-C_{b c a}-C_{c a b}\right) .
$$

$\Sigma_{a b c}$ is also antisymmetric in $a b$, namely $\Sigma_{(a b) c}=0$, and in our case we have

$$
\begin{aligned}
& \Sigma_{\hat{i} \hat{i} \hat{t}}=\Sigma_{\hat{i} \hat{t} \hat{j}}=\frac{1}{2} e^{\phi} \bar{F}_{i j} \\
& \Sigma_{\hat{t} \hat{i} \hat{t}}=D_{i} \phi+\dot{A}_{i}
\end{aligned}
$$

Next one computes the Christoffel symbol defined as usual by

$$
\Gamma_{a b c}=\frac{1}{2}\left(\partial_{b} g_{a c}+\partial_{c} g_{a b}-\partial_{a} g_{b c}\right) .
$$

In our case it is given by

$$
\begin{aligned}
\Gamma_{\hat{j} \hat{k}}^{\hat{i}} & =\bar{\Gamma}[\gamma]_{j k}^{i}[\gamma] \\
\Gamma_{\hat{i} \hat{j}}^{\hat{t}} & =\frac{1}{2} e^{-\phi} \dot{\gamma}_{i j} \\
\Gamma_{\hat{t} \hat{j}}^{\hat{i}} & =\frac{1}{2} e^{-\phi} \gamma^{i k} \dot{\gamma}_{k j},
\end{aligned}
$$

where $\bar{\Gamma}$ is defined by (4.4). Substituting back (A.10, A.12) into (A.6) provides us with the connection $\omega_{a b c}$.

The Einstein-Hilbert action is expressed in terms of the curvature, which is related to derivatives of the connection. However, after integration by parts we found that it could be expressed in terms of the connection only, so there is no need to compute the curvature

$$
(-16 \pi G) S_{E H}=\int R d V \simeq \int \sqrt{g} \theta\left(\omega^{a b c} \omega_{a b c}-\omega_{a} \hat{\omega}^{s}\right)
$$

where $\simeq$ denotes equality up to boundary terms and we define

$$
\begin{aligned}
\theta & :=\prod_{a} \theta^{a} \\
\omega_{a} & :=g^{b c} \omega_{a b c}=g^{b c} \Gamma_{a b c}-C_{a b}{ }^{a} \\
\hat{\omega}_{a} & :=\omega_{b a}^{a}=\Gamma_{b a}^{a}+C_{a b}{ }^{a}
\end{aligned}
$$

We note that if one were to consider $\int f d V R$ where $f$ is any scalar function, the integration by parts would add the term $-\left(\omega^{a}-\hat{\omega}^{a}\right) \partial_{a} \log f$.

Substituting the values of $\omega_{a b c}$ into (A.13), we encounter the expression

$$
-\left(\bar{\Gamma}^{i j k} \bar{\Gamma}_{k i j}-\bar{\Gamma}_{i j}^{j} \gamma^{k l} \bar{\Gamma}_{k l}^{i}+\left(\gamma^{i j} \bar{\Gamma}_{j k}^{k}-\gamma^{k l} \bar{\Gamma}_{k l}^{i}\right)\left(\left(D_{i} \phi+\dot{A}_{i}\right)\right)\right.
$$


where everywhere the connection here is of the spatial metric alone $\Gamma_{i j k}=\Gamma[\gamma]_{i j k}$. Recalling that the measure is $e^{\phi} \sqrt{\widetilde{\gamma}} d^{d-1} x d t$ and using the comment at the end of the last paragraph this expression can be integrated back by parts to yield $-\bar{R}[\gamma]$. Finally replacing back from the temporary notation of this section $\gamma \rightarrow \widetilde{\gamma}$ we obtain the expression for the action (3.3) that we sought to derive.

Harmonic gauge fixing term. In order to calculate this term it is useful to express the harmonic constraint in terms of a hybrid frame formalism. We obtain the following relation

$$
\Gamma_{a}\left[g_{\nu \rho}\right]:=e_{a}^{\mu} \Gamma_{\mu}\left[g_{\nu \rho}\right]=\Gamma_{a}\left[g_{b c}\right]+\Gamma_{a}^{\theta}
$$

where the first term is the usual expression only in terms of the metric in the frame basis

$$
\Gamma_{a}\left[g_{b c}\right]:=g^{b c}\left(\partial_{b} g_{a c}-\frac{1}{2} \partial_{a} g_{b c}\right)
$$

while the second term involves also the frame

$$
\Gamma_{a}^{\theta}:=\left(g_{a b} g^{\nu \rho}+e_{a}^{\rho} e_{b}^{\nu}-e_{a}^{\nu} e_{b}^{\rho}\right) \partial_{\nu} \theta_{\rho}^{b}
$$

\section{B. NRG action expanded}

In this section we present expressions for the expanded action and group it according to the number of time derivatives for the purposes of the PN approximation. These expressions were computed using a rather different method (relying on a presentation of the metric by an orthonormal frame) and were confirmed to be identical with the expanded form of (3.10).

Since each term in the scalar curvature contains precisely two derivatives of the metric with respect to either a spatial or a temporal coordinate, one can organize all the terms into three groups according to the number of temporal derivatives

$$
S_{E H}(g)=\frac{1}{16 \pi G} \int R[g] \rightarrow S^{(0)}(\gamma, A, \phi)+S^{(1)}(\gamma, A, \phi)+S^{(2)}(\gamma, A, \phi) .
$$

We find

$$
\begin{array}{r}
S^{(0)}(\gamma, A, \phi)=-\frac{1}{16 \pi G} \int d^{d} x \sqrt{\gamma}\left[-R[\gamma]+\frac{d-2}{d-3}(\partial \phi)^{2}-\frac{1}{4} e^{2(d-2) \phi /(d-3)} F^{2}\right] \\
S^{(1)}(\gamma, A, \phi)=\frac{1}{16 \pi G} \int d^{d} x \sqrt{\gamma}\left[-\frac{1}{2} e^{2(d-2) \phi /(d-3)} F^{i j} \dot{A}_{[i} A_{j]}+\frac{\partial_{t}\left(\gamma \gamma^{i j}\right)}{\gamma} \nabla_{i} A_{j}\right. \\
\left.-2 \frac{d-2}{d-3} \nabla^{i} \phi\left(\dot{A}_{i}+\dot{\phi} A_{i}\right)\right],
\end{array}
$$

where a dot denotes the derivative with respect to time, the metric $\gamma$ is being used to raise and lower the indices such as $(\partial \phi)^{2}=\gamma^{i j} \partial_{i} \phi \partial_{j} \phi$ and we use the standard definitions $F^{2}=F_{i j}$ and $F^{i j}, \quad F_{i j}=\partial_{i} A_{j}-\partial_{j} A_{i}$. Finally, $S^{(2)}(\gamma, A, \phi)$ is given by 


$$
\begin{aligned}
S^{(2)}(\gamma, A, \phi) & =\frac{1}{16 \pi G} \int d^{d} x \sqrt{\gamma} \frac{1}{4}\left(A^{2}-e^{-2(d-2) \phi /(d-3)}\right)\left(\left(\gamma^{i j} \dot{\gamma}_{i j}\right)^{2}+\dot{\gamma}^{i j} \dot{\gamma}_{i j}\right) \\
& -\frac{1}{16 \pi G} \int d^{d} x \sqrt{\gamma} \frac{1}{2}\left[\dot{\gamma}^{i j} \dot{\gamma}_{i k} A_{j} A^{k}+\gamma^{k l} \dot{\gamma}_{k l} \dot{\gamma}_{i j} A^{i} A^{j}\right] \\
& +\frac{1}{16 \pi G} \int d^{d} x \sqrt{\gamma} \frac{1}{2}\left[\gamma^{i j} \dot{A}_{i} \dot{A}_{j} A^{2}-\left(A^{i} \dot{A}_{i}\right)^{2}\right] e^{2(d-2) \phi /(d-3)} \\
& -\frac{1}{16 \pi G} \frac{d-2}{d-3} \int d^{d} x \sqrt{\gamma} \dot{\phi}^{2}\left[A^{2}+\frac{d-1}{d-3} e^{-2(d-2) \phi /(d-3)}\right] \\
& +\frac{1}{16 \pi G} \frac{d-2}{d-3} \int d^{d} x \sqrt{\gamma} e^{-2(d-2) \phi /(d-3)} \dot{\phi} \gamma^{i j} \dot{\gamma}_{i j} \\
& +\frac{1}{16 \pi G} \int d^{d} x \sqrt{\gamma}\left[\gamma^{k l} \dot{\gamma}_{k l} A^{j} \dot{A}_{j}+\dot{\gamma}^{i j} \dot{A}_{i} A_{j}\right] \\
& -\frac{1}{8 \pi G} \frac{d-2}{d-3} \int d^{d} x \sqrt{\gamma} \dot{\phi} A^{i} \dot{A}_{i},
\end{aligned}
$$

with $A^{2}=\gamma^{i j} A_{i} A_{j}$.

\section{References}

[1] I. Newton, "Philosophice Naturalis Principia Mathematica" (1687).

[2] A. Einstein, "Die Feldgleichungen der Gravitation", Sitzungsber. Preuss. Akad. Wiss. Berlin, 844 (25 Nov 1915).

[3] H. A. Lorentz and J. Droste. English translation in Lorentz Collected papers, Vol 5, 330 (1934-1939), The Hague: Nijhoff.

[4] A. Einstein, L. Infeld and B. Hoffmann, Annals Math. 39, 65 (1938).

[5] V. A. Fock, "The theory of space, time and gravitation", English translation New York: Pergamon Press (1959).

L. Infeld, "Motion and relativity", Oxford : Pergamon Press (1960).

[6] L. Blanchet, "Gravitational radiation from post-Newtonian sources and inspiralling compact binaries," Living Rev. Rel. 5, 3 (2002), update: Living Rev. Rel. 9, 4 (2006)

[arXiv:gr-qc/0202016]. "Post-Newtonian theory and the two-body problem," arXiv:0907.3596 [gr-qc].

[7] G. Schaefer, "Post-Newtonian methods: Analytic results on the binary problem," arXiv:0910.2857 [gr-qc].

[8] L. Blanchet and T. Damour, "Post Minkowskian iteration method and the structure of radiative gravitational fields," C. R. Seances Acad. Sci. II 298, 431 (1984).

[9] L. Blanchet and T. Damour, "PostNewtonian generation of gravitational waves," Annales Poincare Phys. Theor. 50, 377 (1989).

[10] A. Abramovici et al., "LIGO: The Laser Interferometer Gravitational Wave Observatory," Science 256, 325 (1992). B. Caron et al., "The Virgo interferometer," Class. Quant. Grav. 14, 1461 (1997). B. Willke et al., "The GEO 600 gravitational wave detector," Class. Quant. Grav. 19, 1377 (2002). H. Tagoshi et al. [TAMA Collaboration], "The first search for 
gravitational waves from inspiraling compact binaries using TAMA300 data," Phys. Rev. D 63, 062001 (2001) [arXiv:gr-qc/0012010]. A. F. Garcia Marin, G. Heinzel and K. Danzmann, "LISA And Its Precursor Lisa Pathfinder," PoS HEP2005, 036 (2006).

[11] W. D. Goldberger and I. Z. Rothstein, "An effective field theory of gravity for extended objects," Phys. Rev. D 73, 104029 (2006) [arXiv:hep-th/0409156]. W. D. Goldberger, "Les Houches lectures on effective field theories and gravitational radiation," arXiv:hep-ph/0701129.

[12] T. Damour and G. Esposito-Farese, "Testing gravity to second postNewtonian order: A Field theory approach," Phys. Rev. D 53, 5541 (1996) [arXiv:gr-qc/9506063].

[13] B. Kol and M. Smolkin, "Classical Effective Field Theory and Caged Black Holes," Phys. Rev. D 77, 064033 (2008) [arXiv:0712.2822 [hep-th]].

[14] B. Kol and M. Smolkin, "Non-Relativistic Gravitation: From Newton to Einstein and Back," Class. Quant. Grav. 25, 145011 (2008) [arXiv:0712.4116 [hep-th]].

[15] G. Schaefer, "Gravitomagnetic Effects," Gen. Rel. Grav. 36, 2223 (2004) [arXiv:gr-qc/0407116].

[16] J. B. Gilmore and A. Ross, "Effective field theory calculation of second post-Newtonian binary dynamics," Phys. Rev. D 78, 124021 (2008) [arXiv:0810.1328 [gr-qc]].

[17] S. Foffa, R. Sturani, "Effective field theory calculation of conservative binary dynamics at third post-Newtonian order," [arXiv:1104.1122 [gr-qc]].

[18] B. Kol and M. Smolkin, "Dressing the Post-Newtonian two-body problem and Classical Effective Field Theory," Phys. Rev. D 80, 124044 (2009) [arXiv:0910.5222 [hep-th]].

[19] R. A. Porto, A. Ross and I. Z. Rothstein, arXiv:1007.1312 [gr-qc]. M. Levi, arXiv:1006.4139 [gr-qc]. D. L. Perrodin, arXiv:1005.0634 [gr-qc]. R. A. Porto, Class. Quant. Grav. 27, 205001 (2010) [arXiv:1005.5730 [gr-qc]]. D. Brizuela and G. Schaefer, Phys. Rev. D 81, 084014 (2010) [arXiv:1002.4552 [gr-qc]]. W. D. Goldberger and A. Ross, Phys. Rev. D 81, 124015 (2010) [arXiv:0912.4254 [gr-qc]]. J. B. Gilmore, A. Ross and M. Smolkin, JHEP 0909, 104 (2009) [arXiv:0908.3490 [hep-th]]. C. R. Galley and M. Tiglio, Phys. Rev. D 79, 124027 (2009) [arXiv:0903.1122 [gr-qc]]. U. Cannella and R. Sturani, arXiv:0808.4034 [gr-qc]. V. Cardoso, O. J. C. Dias and P. Figueras, Phys. Rev. D 78, 105010 (2008) [arXiv:0807.2261 [hep-th]]. B. Kol, Gen. Rel. Grav. 40, 2061 (2008) [Int. J. Mod. Phys. D 17, 2617 (2009)] [arXiv:0804.0187 [hep-th]]. J. Le Witt and S. F. Ross, JHEP 1001, 101 (2010) [arXiv:0910.4332 [hep-th]]. R. Emparan, T. Harmark, V. Niarchos and N. A. Obers, JHEP 1003, 063 (2010) [arXiv:0910.1601 [hep-th]]. B. Kleihaus, J. Kunz, E. Radu and C. Stelea, JHEP 0909, 025 (2009) [arXiv:0905.4716 [hep-th]]. M. Headrick, S. Kitchen and T. Wiseman, Class. Quant. Grav. 27, 035002 (2010) [arXiv:0905.1822 [gr-qc]]. R. Emparan, T. Harmark, V. Niarchos and N. A. Obers, Phys. Rev. Lett. 102, 191301 (2009) [arXiv:0902.0427 [hep-th]]. Y. Z. Chu, Phys. Rev. D 79, 044031 (2009) [arXiv:0812.0012 [gr-qc]].

[20] T. Kaluza, "Zum Unitätsproblem in der Physik". Sitzungsber. Preuss. Akad. Wiss. Berlin. (Math. Phys.) 1921: 966972. O. Klein, "Quantentheorie und fünfdimensionale Relativitätstheorie". Zeitschrift für Physik A Hadrons and Nuclei 37 (12): 895906.

[21] R. L. Arnowitt, S. Deser and C. W. Misner, "The dynamics of general relativity," in Gravitation: an introduction to current research, Louis Witten ed. (Wiley 1962), chapter 7, p 227; [arXiv:gr-qc/0405109]. 
[22] E. Gourgoulhon, "3+1 Formalism and Bases of Numerical Relativity," arXiv:gr-qc/0703035.

[23] B. Kol, M. Levi, M. Smolkin, "Comparing space+time decompositions in the post-Newtonian limit," [arXiv:1011.6024 [gr-qc]].

[24] "Gravitomagnetism", Wikipedia the free encyclopedia, http://en.wikipedia.org/wiki/Gravitoelectromagnetism.

[25] R. Wald, "Gravitational spin interaction," Phys. Rev. D 6, 406 (1972).

[26] B. deWitt, Phys. Rev. 1601113 (1967), eq. (6.6).

[27] C. S. Aulakh and D. Sahdev, "The Infinite Dimensional Gauge Structure Of Kaluza-Klein Theories. 1. D = 1+4," Phys. Lett. B 164, 293 (1985).

[28] A. Maheshwari, "A Study Of The Higgs Effect In The Five-Dimensional Kaluza-Klein Theory," Pramana 27, 383 (1986).

[29] M. J. Duff, B. E. W. Nilsson and C. N. Pope, "Kaluza-Klein Supergravity," Phys. Rept. 130, 1 (1986). J. M. Overduin and P. S. Wesson, "Kaluza-Klein gravity," Phys. Rept. 283, 303 (1997) [arXiv:gr-qc/9805018].

[30] C. Misner, K. Thorne and J. Wheeler, "Gravitation," Ch. 14.6 Freeman (1973). 